International Journal of Social Psychiatry2021

\title{
Analysis of the early mother-child relationship in schizophrenic patients
}

\section{( 国 Article in press ? )}

- Khoshgoftar, M.a,

- Khodabakhshi-Koolaee, A. ${ }^{a}$ Email Author,

- Sheikhi, M.R. ${ }^{\mathrm{b}}$

- aDepartment of Psychology and Educational Sciences, Faculty of Humanities, Khatam University, Tehran, Iran

- 'social Determinants of Health Research Center, Research Institute for Preventio $\mathrm{n}$ of Non-Communicable Diseases, Qazvin University of Medical Sciences, Qazvin, Iran

\begin{abstract}
View references (25)
Background and Aim: The mother as the first caregiver plays a significant role in the formation of the child's behavior, growth, and communication. The present study aimed to analyze the early mother-child relationship in schizophrenic patients. Materials and Methods: This qualitative study employed a descriptive phenomenological approach. The participants were male patients with schizophrenia who were hospitalized in Qazvin Bahman Psychiatric Hospital from March to September 2020 with an age range of over 18 years. Given the objective of the study, the data were collected using semistructured interviews. The participants were selected using purposive sampling and the sampling procedure continued until data saturation as the point when no new information is observed in the data. Accordingly, the data were saturated after interviewing 15 participants. The data were analyzed using Colaizzi's seven-step method. Results: The analysis of the data revealed four main themes including ambivalent attachment to the mother, feelings of constant fear and worry, a sense of constant care for the mother, and a cold and emotionless relationship with the child. Conclusion: The present study suggested that schizophrenia is a disorder that affects the mother-child relationship, and does the term "schizophrenic mothers" need to be reconsidered? However, the result of this research has been done according to the nature and cultural context of Iranian society. (C) The Author(s) 2021.
\end{abstract}

